\title{
Rotura del tendón del pectoral mayor: técnica de reparación abierta durante periodo agudo
}

\section{Pectoralis Major Tendon Rupture: Open Repair Technique during Acute Period}

\author{
Nicolás Morán Córdova ${ }^{1}$ José Laso Errázuriz ${ }^{2}$ Valentina Arias Acuña ${ }^{3}$ Patricio Melean Quiroga ${ }^{4}$ \\ ${ }^{1}$ Especialista en Traumatología y Ortopedia, Universidad de Los \\ Address for correspondence Nicolás Morán Córdova, Traumatología, \\ Andes, Hospital del Trabajador - ACHS, Santiago, Chile \\ 2 Becado de Traumatología y Ortopedia, Universidad de Chile, \\ Hospital del Trabajador - ACHS, Santiago, Chile \\ ${ }^{3}$ Médico Cirujano, Facultad de Medicina, Universidad de los Andes, \\ Santiago, Chile \\ ${ }^{4}$ Cirujano de Hombro, Práctica Privada, Santiago, Chile \\ Rev Chil Ortop Traumatol 2018;59:110-116. \\ Cirujano de Hombro, Hospital del Trabajador - ACHS, Ramón Carnicer \\ 185, Providencia, Santiago, Chile (e-mail: nimoran@miuandes.cl).
}

\section{Resumen \\ Palabras claves \\ - tendón pectoral mayor \\ - rotura tendón \\ - reparación pectoral mayor}

La rotura del tendón del pectoral mayor es considerada una lesión infrecuente en lo descrito en la literatura, pero esa lesión ha tenido un aumento exponencial en sus reportes a partir de los años 90 . A continuación, presentamos la resolución quirúrgica de una rotura completa del tendón del pectoral mayor mediante una técnica simple y reproducible en un hombre de 34 años, sometido a una alta demanda física diaria. Tenemos ya publicado diferentes técnicas de reparación en periodo agudo como reconstrucción en lesiones crónicas. Nosotros presentamos una técnica de reparación para rotura aguda que consiste en la reinserción mediante anclas de titanio en su huella insercional mediante una configuración de suturas que forma un constructo estable y resistente, que permita al paciente rehabilitarse en forma precoz y satisfactoria para intentar volver al mismo nivel de actividad pre lesional. Con eso, aportamos al especialista una manera sencilla de enfrentarse y planificar la reparación de ese tipo de lesiones infrecuentes que no siempre estamos acostumbrados a resolver.

Pectoralis major tendon rupture is an infrequent injury in what has been described in the literature so far. Lately, an exponential increase in reported cases of this injury has been observed. We present a simple and reproducible surgical technique in a 34 years old male subject to a high daily physical demand. Different techniques have been published for tendon repair during the acute period as well as reconstruction in chronic lesions. We present a technique for acute rupture that consists of the reinsertion by means of titanium anchors in its insertional footprint through a suture configuration that forms a stable and resistant construct, that allows the patient to be rehabilitated in an early and satisfactory manner with the objective to return to the same Level of pre-injury activity. With this we provide the specialist with a simple way to manage the repair of these infrequent injuries. received

March 28, 2018

accepted

December 4, 2018

published online

February 15, 2019
Copyright (e 2018 by Thieme Revinter

Publicações Ltda, Rio de Janeiro, Brazil
License terms

10.1055/s-0039-1677757. ISSN $0716-4548$.

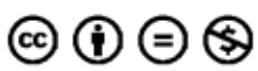




\section{Introducción}

A pesar de ser considerada una lesión infrecuente, la incidencia de rotura del músculo pectoral mayor (PM) va en aumento, demostrado por el número de publicaciones en las últimas décadas. ${ }^{1-3} \mathrm{~A}$ menudo, esos pacientes están sometidos a altas demandas físicas, como un levantador de pesas o población militar activa. De hecho, la única incidencia calculada es en esa última población, la cual fue de 60 por 100,000 persona-año según Balazs y col., ${ }^{2}$ con una tasa de riesgo de lesión de 1 por 13.317 persona-años en la población militar US, según White y col. ${ }^{3}$

El PM es un músculo ancho, compuesto por 2 vientres musculares, cada uno conformado por múltiples haces, presentando una compleja inserción humeral bilaminar, ${ }^{4}$ descrita en un 59-65\% de los casos como la zona de lesión del PM. ${ }^{4,5}$ La clasificación más utilizada para esa lesión es descrita por Tietjen y col. ${ }^{6}$ la cual presenta una utilidad anatomo-topográfica, agrupando las roturas en 3 grados (Tabla 1 y -Fig. 1). Sin embargo, dado la imprecisión anatómica sobre el PM, ElMaraghy ${ }^{4}$ propone una nueva clasificación que sería homologable a las roturas del manguito rotador, al agruparlas según el espesor: parciales o totales, y según el ancho: completas e incompletas, con diferentes combinaciones y recomendaciones en su tratamiento (-Fig. 2).

Varios estudios concluyen que el diagnóstico precoz y el tratamiento agudo, favorecen los resultados exitosos. ${ }^{5,7,8}$ Para ello, se requiere de una comprensión adecuada de la historia clínica para reconocer el tipo de paciente y mecanismo habitual, por ejemplo, levantador de pesa realizando press banca quien sufre contracción excéntrica del PM con el codo extendido. ${ }^{8}$ El diagnóstico es apoyado por un examen físico y estudio imagenológico quienes tendrán mayor precisión en aquellas roturas que sean: completas, agudas y de localización tendón-hueso. ${ }^{9}$ Aunque no es un músculo requerido para la mayoría de las actividades básicas, se recomienda ser reparado

Tabla 1 Clasificación de Tietjen ${ }^{6}$ para roturas del músculo PM

\begin{tabular}{|c|c|c|c|}
\hline Tipo & Subtipo & Descripción & $\begin{array}{l}\text { Tratamiento } \\
\text { recomendado }\end{array}$ \\
\hline I & Contusión & & $\begin{array}{l}\text { Conservador } \\
\text { (no quirúrgico) }\end{array}$ \\
\hline II & $\begin{array}{l}\text { Rotura } \\
\text { parcial }\end{array}$ & & $\begin{array}{l}\text { Conservador } \\
\text { (no quirúrgico) }\end{array}$ \\
\hline \multirow[t]{5}{*}{ III } & $\begin{array}{l}\text { Rotura } \\
\text { completa }\end{array}$ & & \\
\hline & A & $\begin{array}{l}\text { Origen } \\
\text { esternoclavicular }\end{array}$ & $\begin{array}{l}\text { Conservador } \\
\text { (no quirúrgico) }\end{array}$ \\
\hline & B & $\begin{array}{l}\text { Vientre } \\
\text { muscular }\end{array}$ & $\begin{array}{l}\text { Conservador } \\
\text { (no quirúrgico) }\end{array}$ \\
\hline & C & $\begin{array}{l}\text { Unión } \\
\text { miotendínea }\end{array}$ & $\begin{array}{l}\text { Quirúrgico en } \\
\text { compromiso } \\
\text { funcional / } \\
\text { estético severo }\end{array}$ \\
\hline & $\mathrm{D}$ & Inserción & Quirúrgico \\
\hline
\end{tabular}

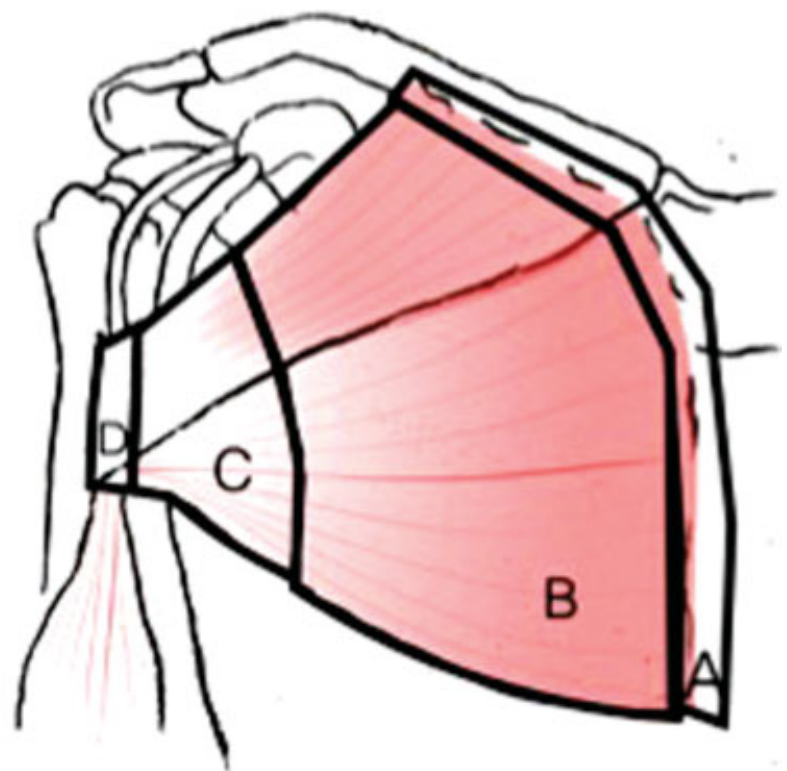

Fig. 1 Imagen representativa de la clasificación de Tietjen ${ }^{6}$ para roturas del tendón del PM.

en aquellos paciente jóvenes sometidos a altas demandas físicas, con roturas completas en la unión miotendínea como insercional, ya que su reinserción quirúrgica otorga los mejores resultados funcionales, cosméticos y de satisfacción. ${ }^{1,3-5,7}$ Con respecto a la configuración en la técnica de reparación, no ha sido demostrado la superioridad clínica de una sobre la otra, contando solo con estudios pre clínicos que buscan beneficios biomecánicos que no necesariamente concuerdan con mejores resultados funcionales. ${ }^{10,11}$

El objetivo principal de este artículo es presentar la resolución quirúrgica de una rotura completa del tendón del pectoral mayor, aguda, mediante una técnica simple y reproducible en un hombre de 34 años sometido a una alta demanda física. Nuestro objetivo secundario, es realizar una revisión de la literatura sobre esa lesión para complementar la discusión del caso.
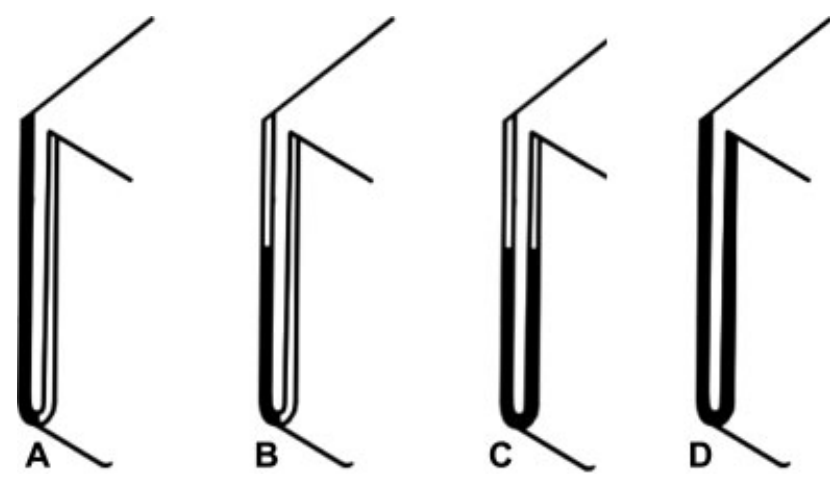

Fig. 2 Clasificación de ElMaraghy ${ }^{4}$ (adaptada) para roturas del tendón PM. Espesor que se refiere al compromiso de una o ambas porciones del músculo (clavicular y esternal), y el ancho que hace referencia al compromiso en el eje transversal del músculo. De izquierda a derecha; (A) espesor parcial-ancho total; (B) espesor parcial-ancho parcial; (C) espesor total-ancho parcial; (D) espesor total-ancho total. 


\section{Caso Clínico}

Paciente de 34 años género masculino, sano, profesor de educación física, no usuario de anabólicos, al elevar plato de pesas de $50 \mathrm{~kg}$ sobre el nivel de la cabeza, éste cae hacia posterior provocando dolor brusco en cara anterior del hombro derecho, evolucionando con impotencia funcional.

Al examen físico se constata dolor a la palpación de cara anterior del brazo derecho que irradia a pared torácica, aumento de volumen en área del PM derecho, equimosis pre bicipital y pérdida de la línea axilar anterior asociado a déficit de rango en aducción y rotación interna. Estudio radiológico descarta lesión ósea aguda, sin embargo, la ecografía de partes blandas del brazo y pectoral mayor informó desgarro completo de la inserción humeral del músculo PM derecho. Al complementar el estudio con Resonancia Magnética (RM) de hombro, el informe no confirmó la presencia de rotura del PM, y en las imágenes, se logró visualizar sólo rotura de la cabeza clavicular del PM con mínima retracción medial (- Fig. 3). Dado la alta sospecha de rotura completa, se decide realizar exploración quirúrgica.

\section{Técnica Quirúrgica}

Paciente con anestesia general más bloqueo interescalénico, en posición silla de playa "low" ( $40^{\circ}$ de inclinación), ocupando posicionador espacial de extremidad TRIMANO (Arthrex, Naples, Florida).

Se realiza un abordaje deltopectoral de $5 \mathrm{~cm}$ más caudal de lo habitual directo a la inserción humeral del PM. Se protege la vena cefálica hacia lateral durante todo el procedimiento. Se realiza una disección prolija hasta identificar la zona de inserción humeral del tendón del PM, el cual estaba completamente desinsertado, tanto la cabeza esternocostal como clavicular, ambas retraidas en forma moderada. Posteriormente, se realiza una liberación de ambos cabos tendíneos del PM hasta lograr reducirlos a su posición lateral en la cresta bicipital.

Se procede a realizar una cruentación del footprint humeral con cucharilla y con fresa redonda tipo "pimpollo," dibujando un área longitudinal que corresponde a la zona de inserción, con el objeto de mejorar la biología local y conseguir una mejor cicatrización.

A continuación, se brocan tres túneles de $3,5 \mathrm{~mm}$ de diámetro en distribución lineal céfalo caudal para insertar anclas Corkscrew 5,5 Titanio doble carga de suturas de alta resistencia Fiberwire 2 (Arthrex, Naples, Florida). Ese brocado previo permite insertar las anclas Corkscrew de manera segura, evitando así violar la cortical y producir fracturas en el área de inserción.

Luego, a partir de las dos anclas más cefálicas, se pasan 2 puntos con configuración Mason-Allen modificado al haz esternal (eje horizontal), dejando las colas de las suturas largas (- Fig. 4). Con el ancla más caudal, se sutura un nuevo Mason-Allen modificado al haz clavicular (eje vertical), y se refuerza con 2 Mason-Allen modificados usando las colas remanentes de las 2 anclas más cefálicas, logrando una reparación anatómica sin tensión y una aposición de ambos cabos tendíneos de forma adecuada (-Figs. 5 y 6 ).

\section{Cuidado Post Operatorio y Rehabilitación}

Se indicó el uso de inmovilizador de hombro de manera estricta por 6 semanas, acompañado de ejercicios pendulares desde el 1er día post operatorio, evitando la abducción y rotación externa forzada. Los rangos de movimiento (ROM) pasivos se iniciaron a partir de la sexta semanas, ROM activos a las 10 semanas, y finalmente fortalecimiento muscular a partir de las 12 semanas post reparación.
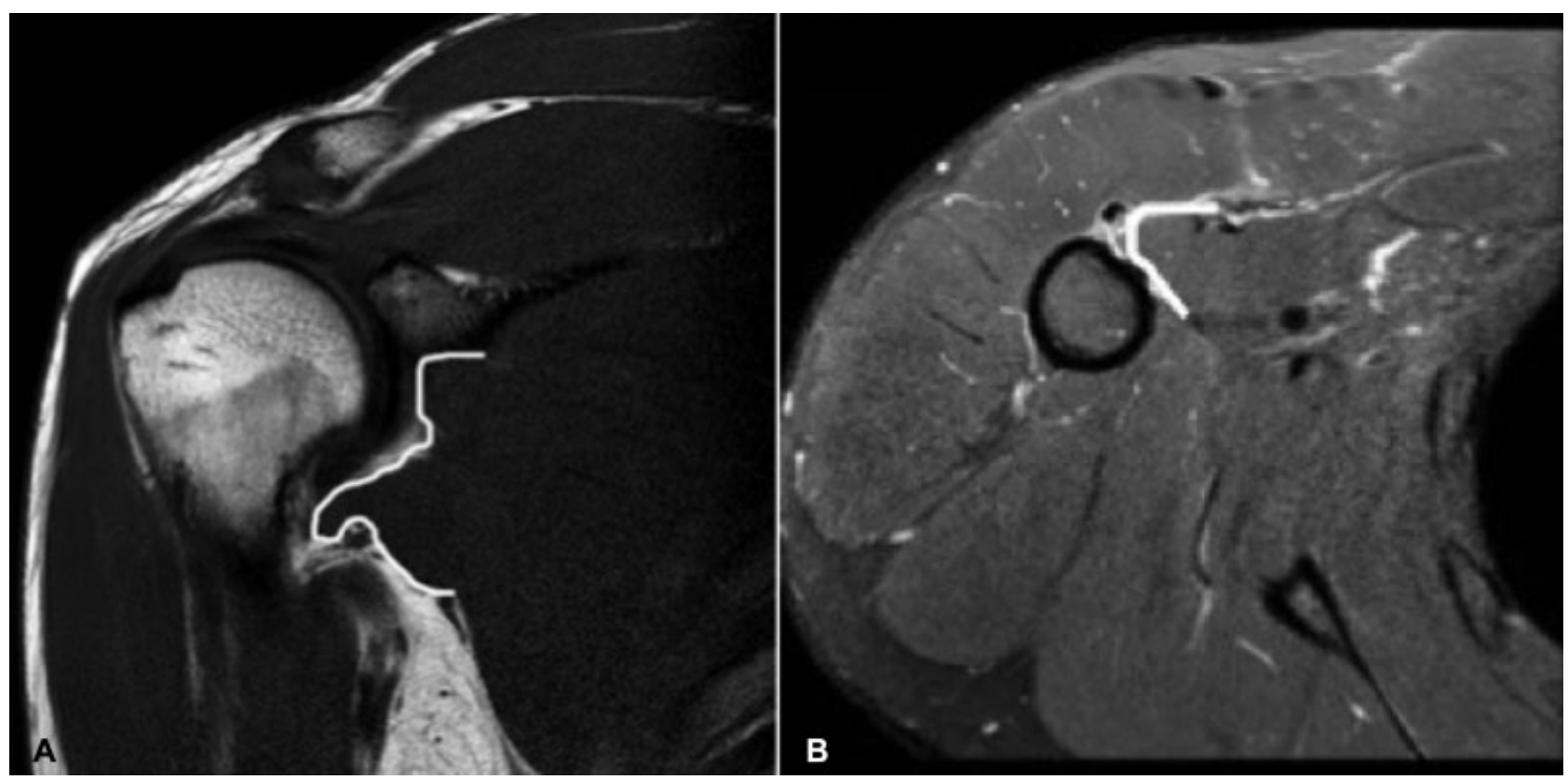

Fig. 3 RM de hombro derecho (A) Corte coronal de secuencia T1, evidencia retracción de las fibras inferiores del pectoral mayor, con ligera ondulación de las mismas. (B) Corte axial de secuencia T2 que presenta discontinuidad de las fibras del PM a nivel de su inserción humeral, asociado a leve edema. Los rebordes tendíneos rotos del PM se delinearon de color blanco. 


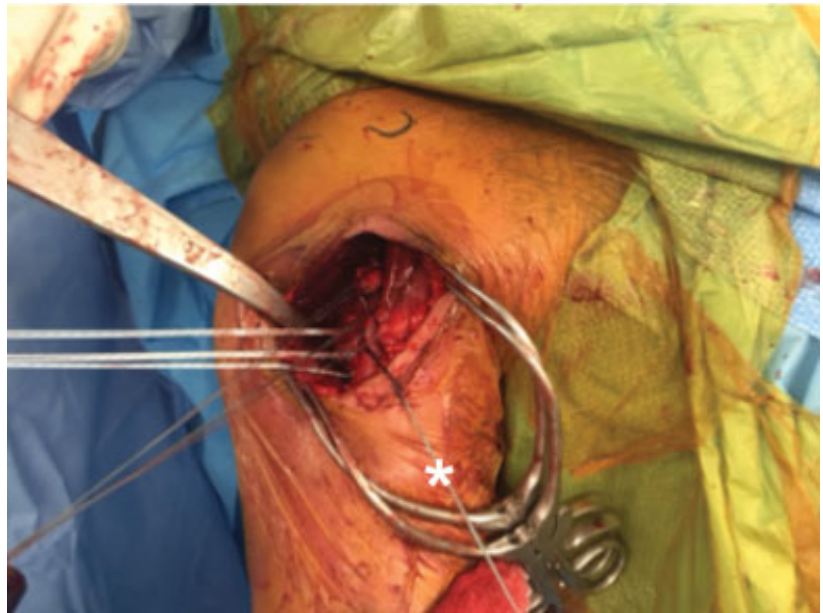

Fig. 4 Imagen clínica que muestra la porción esternal del pectoral mayor ya en el footprint con las dos suturas tipo Mason Allen modificado y una sutura libre marcadas con un asterisco $\left({ }^{*}\right)$.

\section{Evaluación Final}

El paciente evolucionó favorablemente, sin complicaciones postoperatorias inmediatas ni a mediano plazo. La radiografía de control mostró óptima posición de las anclas (-Fig. 7). Nuestro paciente, entrenador de Cross fit, logró un reintegro laboral a los 4 meses y retorno al mismo nivel pre-lesión a los 6 meses, con un score de 0 en la escala visual análoga (EVA) del dolor, valoración subjetiva del hombro (SSV) $100 \%$ y un Constant post operatorio de 95 puntos al término del seguimiento (10 meses).

Según los criterios de Bak, ${ }^{5}$ el paciente fue clasificado como excelente dentro sus resultados: libre de dolor, rangos de movimientos completos, sin problemas cosméticos, sin déficit de aducción y un retorno al mismo nivel pre-lesión.

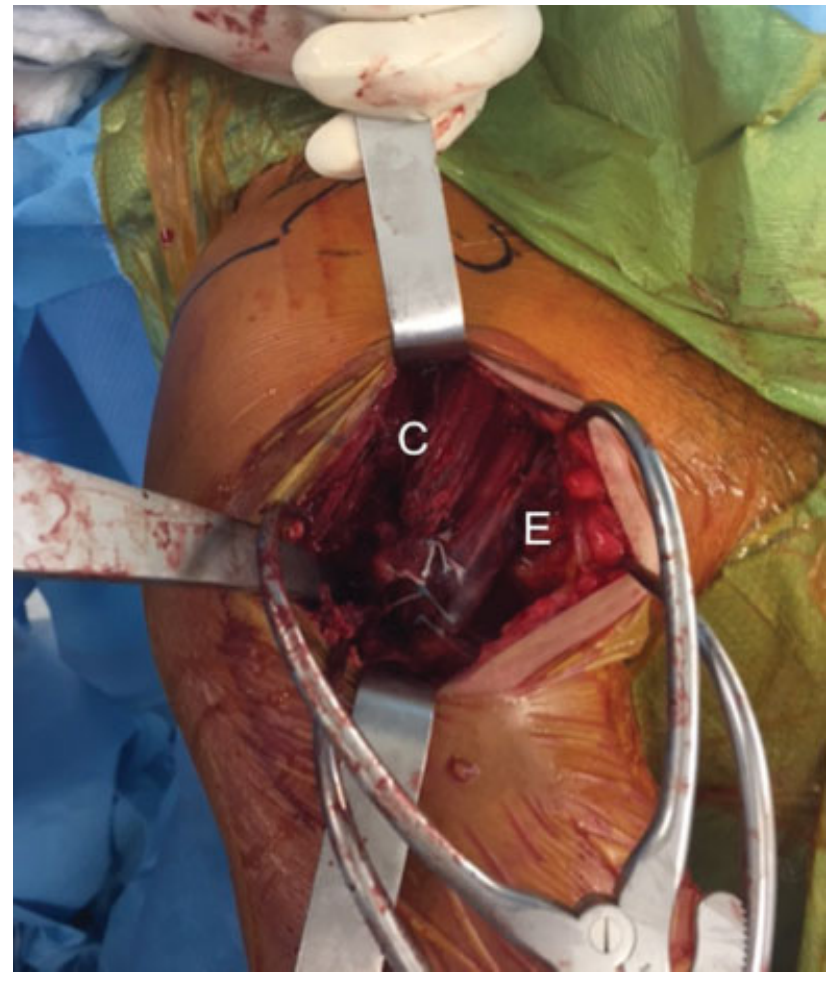

Fig. 6 Se observa la reparación del tendón del PM finalizada de ambos haces musculares del tendón del PM. Esternal (E); clavicular (c).

\section{Discusión}

La anatomía del músculo PM se caracteriza por ser compleja, y el conocimiento acabado de la misma resulta fundamental para comprender sus tipos de lesión y eventual reparación. ${ }^{1,4,5,8}$

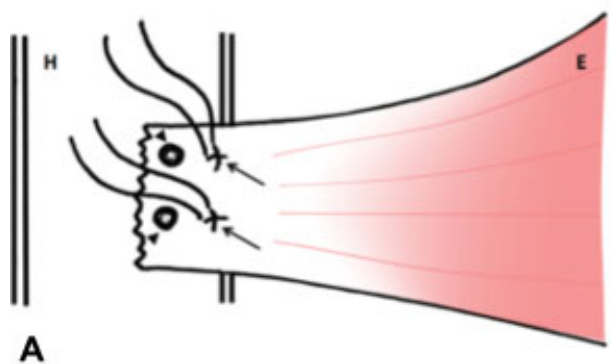

A

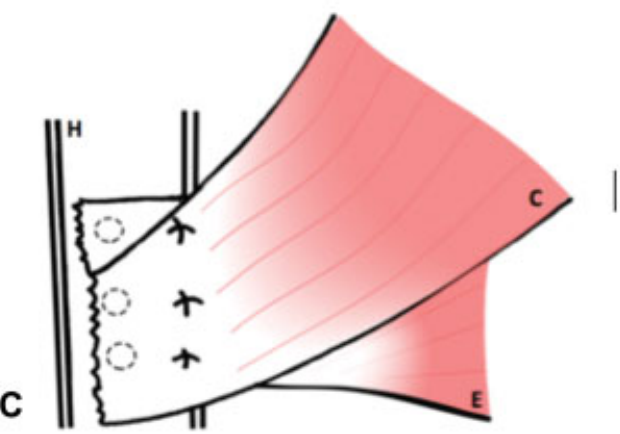

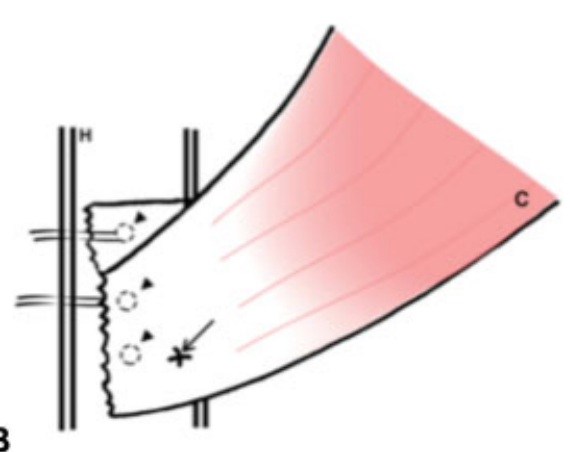

B

Fig. 5 Dibujo esquemático de la reparación. (A) Inserción con anclas y sutura tipo Mason-Allen en la porción esternal del tendón del PM. (B) Reducción de la porción costal del tendón del PM. (C) Fijación final de ambos haces del tendón del PM. (E: porción esternal, C: porción clavicular; H: húmero). 


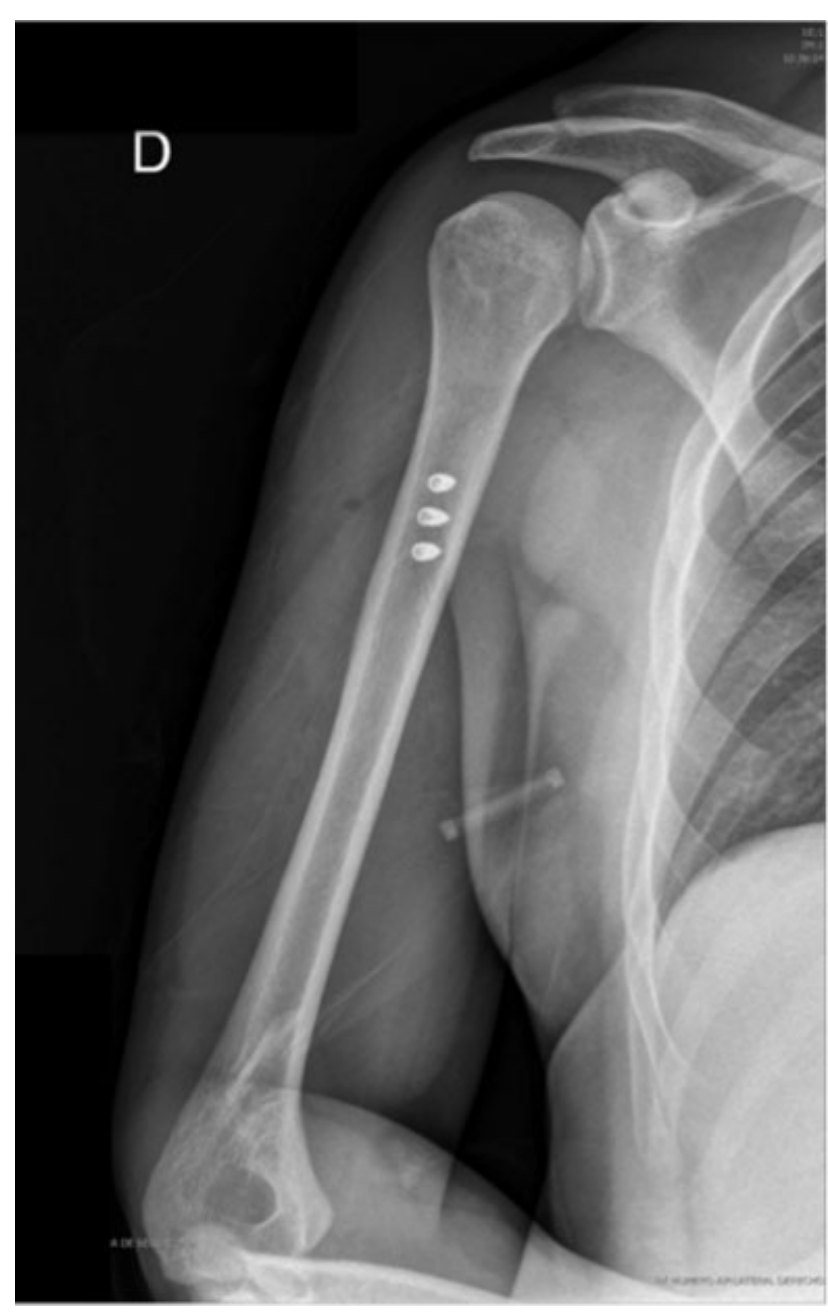

Fig. 7 Radiografía de húmero derecho anteroposterior durante control postoperatorio. Se observa la alineación céfalo caudal de las 3 anclas a nivel diafisiario.

El PM presenta dos cabezas con diferentes orígenes y una inserción común aún más compleja. ${ }^{4}$ La cabeza clavicular tiene su origen en el tercio medial de la clavícula, con una dirección oblicua de sus fibras hacia caudal. La cabeza esternocostal corresponde al $80 \%$ de la masa muscular total, cuyo origen se encuentra tanto en el margen costal esternal, desde la 2da a 6ta costilla y fascia del músculo oblicuo externo. A diferencia de la cabeza clavicular, sus fibras se distribuyen en una dirección más horizontal.

La inserción humeral de ambos cabos se encuentra lateral al surco bicipital, 4 a $5 \mathrm{~cm}$ distales al vértice de la tuberosidad mayor, donde convergen las fibras de ambas cabezas formando un estructura bilaminar en forma de "U," compuesta por una capa antero-inferior principalmente por fibras de la cabeza clavicular y parte de los segmentos superiores de la esternocostal; y otra capa postero-superior, por fibras solo de la cabeza esternocostal, donde las más inferiores terminan en una dirección superolateral. ${ }^{12}$ Esas características le permiten ser un potente aductor y rotador interno, así como un débil elevador del hombro.

El PM, al presentar diferente longitud de sus fibras musculares en los distintos vientres musculares, provoca que la excursión de ellas sea diferente, dando como resultado que la producción de energía se maximice sobre una amplia gama de movimientos, pero que aumente el riesgo de rotura en posiciones desfavorables para su máxima excursión. Según Wolfe y col., ${ }^{13}$ como las fibras musculares inferiores logran habitualmente mayor excursión que las superiores, eso podría explicar por qué las roturas presentan una secuencia predecible desde cefálico hacia caudal.

Las roturas del PM ameritan ser reportadas en la literatura nacional dado su baja y desconocida incidencia en la población general. El caso clínico presenta algunas características epidemiológicas de esta lesión: hombre entre 30-50 años, deportista levantador de pesas, que durante un mecanismo de contracción excéntrica sufre la rotura del PM. ${ }^{1-3}$ El examen físico de ese tipo de lesión aguda puede mostrar un abultamiento y equimosis a nivel torácico, axilar e incluso del brazo, con una pérdida tanto del receso axilar anterior como la visualización del tendón al mismo nivel. Concordamos con Haley y col., ${ }^{1}$ que son esos los dos hallazgos clínicos más precisos de rotura completa del PM, presentes en nuestro paciente, al pedírsele que realizará la aducción de ambas extremidades con manos en ambas crestas iliacas, confirmando la sospecha. Otra manera de reproducirlos, es pidiéndole al paciente que adopte posición de oración y realice contractura de ambos músculos pectorales. Otro hallazgo sugerente de rotura completa es la presencia de un defecto palpable en la misma inserción humeral.

El estudio inicial del paciente fue con radiografías de hombro para descartar lesión ósea aguda, en ese contexto una posible avulsión; además, se realizó una ecografía de partes blandas a nivel del brazo y PM la cual confirmó la rotura completa y sugirió complementar con una RM. En primera instancia, se solicitó RM de hombro con especificación de incluir brazo, pero solo fueron incluidos los cortes de una RM de hombro estándar, por ende, la rotura no fue descrita por el radiólogo, y el cirujano no obtuvo imágenes adecuadas para ver completamente el PM, solo observando ascenso y retracción medial de la cabeza clavicular. Frente a la alta sospecha diagnóstica de rotura completa, tanto por la clínica como la ecografía, se decidió la exploración abierta del músculo, logrando corroborar que ambos vientres musculares estaban desinsertos en forma completa. Eso demuestra que, en algunos casos, el diagnóstico imagenológico puede ser insuficiente, y podemos requerir de una evaluación intraoperatoria para el diagnóstico definitivo, sobre todo cuando exista una alta sospecha clínica. Los estudios que proponen el uso de RM para el diagnóstico de roturas del PM describen protocolos imagenológicos que abarcan parte del hemitórax para incluir completamente al músculo, como lo refiere Chang y col., ${ }^{9}$ cuyos reparos topográficos de referencia pueden ir desde el esternón al húmero (eje medial-lateral), y desde la articulación acromioclavicular al tercio medio de la diáfisis humeral (eje cefalocaudal). De manera retrospectiva, posterior a la reparación abierta del PM, se analizaron los cortes de la RM donde se lograron evidenciar los cabos tendíneos. Destacamos, que los rebordes tendíneos rotos no presentaron imágenes de edema que ayudaran al diagnóstico. 
Carrino y col., ${ }^{14}$ destacó que las mejores secuencias para evaluar las roturas agudas del PM serían STIR y T2 con supresión grasa para visualizar mejor el edema alrededor de la rotura; el corte axial es quien proporciona mayor información, y luego sería el coronal oblicuo. Además, sugiere que, realizando el examen con el hombro en abducción y rotación externa, la capacidad de detectar lesiones aumentaría por la tensión de las fibras musculares que provoca una distracción del defecto.

La sensibilidad (S) y especificidad (E) de la RM para el diagnóstico de roturas del PM fue estudiada por el mismo Chang y col., ${ }^{9}$ quien concluye que la mayor $\mathrm{S}$ y valor predictivo positivo fue en aquellas roturas agudas (S 0,85 para haz esternal - 0,88 para haz clavicular), completas ( $\mathrm{S}$ 1,00 en ambas cabeza) y en la unión tendón hueso (S 0,93 esternal - 0,9 clavicular), en comparación al gold standard que es la evaluación intraoperatoria.

El uso de ecografía fue estudiado por Lee y col., ${ }^{15}$ quien concluyó que el rendimiento diagnóstico para detectar avulsiones del tendón del PM puede verse alterado por la acumulación de fluido laminar en la unión miotendínea, el cual podría llevar a interpretarlo como una rotura y no una avulsión.

En nuestro conocimiento, no se ha comparado el rendimiento diagnóstico de la ecografía con la RM, sin embargo, ninguno de esos exámenes está libre de falsos negativos. Por lo tanto, recomendamos que el estudio y tratamiento final deban orientarse según la sospecha clínica.

Para el manejo de ese tipo de lesiones, aquellas contusiones, roturas parciales, a nivel muscular $\mathrm{u}$ origen, paciente sedentario, deben ser manejadas en forma conservadora. En pacientes jóvenes, sometidos a altas demandas físicas, con roturas completas en la unión miotendínea como insercional, la indicación de reparación quirúrgica es lo más aconsejado en la práctica. ${ }^{1-5,13}$

A pesar que la literatura descrita referente a esa patología aún es controversial, en relación a la temporalidad de la lesión, ellas se dividen en agudas o crónicas. ${ }^{1,4,5,16-18}$ Consideramos que a partir de las 3 semanas se puede encontrar una retracción significativa lo que puede aumentar el grado de disección y complejidad de la cirugía, sin embargo, a las 6 semanas, resulta más aceptable definirla como rotura crónica, dado que la retracción también iría acompañada de una degeneración muscular, sin existir estudios histopatológicos que avalen lo recientemente descrito. Esa definición nos permite comparar los resultados entre roturas agudas vs crónicas, en la cual aquellas reparaciones agudas han presentado mejores resultados funcionales, alivio del dolor, fuerza y satisfacción. $5,7,16$

Se han descrito varias técnicas de reparación en periodo agudo: reinserción con túneles óseos, botones, anclas y tenorrafias en la unión miotendínea. Actualmente, no existen estudios clínicos que demuestren superioridad de una técnica sobre otra.

Rabuck y col. ${ }^{11}$ en un estudio cadavérico, demostró diferencias significativas entre túnel óseo y anclas con suturas, siendo superior la técnica con túnel óseo (carga al fallo $=596 \mathrm{~N}$ vs $383 \mathrm{~N}$ respectivamente, $\mathrm{p}=0,007$ ), pero una de las fallas de esa última técnica fue vía fractura del humero proximal, que puede llegar a ser catastrófica en un deportista sometido a cargas rotacionales, por lo que según el autor principal (PM), la reinserción con anclas es una técnica quirúrgica adecuada y suficiente, con la cual no se han descrito fracturas de húmero proximal (nivel de evidencia tipo VI).

Para aquellas lesiones crónicas se describen reconstrucciones con autoinjerto de tendones isquiotibiales, uso de autoinjerto hueso tendón hueso patelar, autoinjerto de fascia lata o aloinjerto aquiliano, entre otros, que no es objetivo analizar en este artículo.

Con respecto a nuestro caso clínico, frente al diagnóstico de una rotura completa de PM en periodo agudo a nivel de la unión hueso tendón (Tietjen tipo IIID), se decidió la reparación mediante anclas de titanio similar a lo publicado por Butt y col., ${ }^{17}$ que consiste en la reinserción de ambas cabezas a nivel de su footprint a través de anclas con suturas anudadas en forma deslizante, en posición y dirección de las fibras musculares de cada vientre muscular: una capa más anterior con fibras en dirección vertical-oblicua (cabeza clavicular), y una más posterior con fibras en dirección horizontal (cabeza esternocostal). ${ }^{5}$ Esa configuración anatómica tiene el objetivo de lograr la mayor resistencia de las anclas, compresión y disminución del gap en su inserción. ${ }^{3}$

Al finalizar la cirugía, es necesario evaluar la seguridad y nula tensión de la reparación para optimizar la rehabilitación y evitar complicaciones. Gregory y col. ${ }^{18}$ evaluó con el uso de botones, como influye la técnica de sutura y material de sutura en la integridad biomecánica de la reparación del PM, al comparar 4 grupos de reparación: Grupo control = sutura tipo Mason-Allen modificado con 3 botones 6 hebras; Grupo Triple $=$ el mismo grupo control, pero con 3 botones 9 hebras; Grupo Configuración = punto Krakow/Bunnel con sutura de alta resistencia (FiberWire; Arthrex, Inc), y Grupo Tape $=$ punto Krakow/Bunnel con cinta de alta resistencia (FiberTape; Arthrex, Inc). Este estudio concluyó que un mayor número de hebras no aumenta la resistencia de la reparación, y que reparación con FiberTape sería la más resistente en términos de carga al fallo (Krakow/Bunnel con FiberTape $726 \mathrm{~N}$ vs Mason-Allen simple $330 \mathrm{~N}$; Mason-Allen triple $400 \mathrm{~N}$; Krakow/Bunnel con Fiberwire $509 \mathrm{~N}$ ), y, además, la mayoría fallaba por pull-out de la sutura. En nuestro caso, a pesar de utilizar uno de los constructos menos resistente desde un punto de vista biomecánico (ancla con sutura de alta resistencia, no tape), el uso del punto Mason-Allen modificado por cada ancla reforzado con las mismas suturas remanentes logró obtener buenos resultados, permitiendo una rehabilitación precoz y reintegro del paciente a sus actividades previas sin complicaciones a mediano plazo. Cabe destacar que, al momento de insertar las anclas, ellas inicialmente fallaron por la densidad ósea del hueso cortical de la diáfisis humeral, por lo cual debió brocarse un diámetro mayor que permitiera su inserción press fit. Uno de los beneficios de usar anclas en comparación a un túnel óseo es la menor incidencia de fractura de húmero, por eso es que recomendamos una separación de al menos $1 \mathrm{~cm}$ entre cada ancla, realizando 
un brocado de 3,5mm para anclas 5,5mm, y así poder evitar una fractura intraoperatoria entre cada ancla.

En la actualidad, no se han descrito en la literatura estudios comparativos entre grupos y técnicas quirúrgicas necesarias para validar los resultados obtenidos dentro del laboratorio en muestras cadavéricas humanas o animales, por lo que para poder definir cuál técnica quirúrgica resultará en mejores resultados a largo plazo, tanto desde un punto de vista estructural como clínico, se necesitan estudios prospectivos randomizados que aporten esa información.

\section{Conclusión}

La alta sospecha diagnóstica y comprensión anatómica del PM resultó ser imprescindible para realizar en forma oportuna su reparación en este caso clínico de rotura aguda traumática. La técnica descrita permitió realizar una rehabilitación óptima que logró un reintegro a las actividades pre-lesión en forma satisfactoria. La RM de hombro estándar fue insuficiente para el estudio de esta lesión, siendo un pilar fundamental la sospecha clínica.

\section{Conflictos de Interés}

El Dr. Patricio Meleán es Editor Asociado de la Revista Chilena de Ortopedia y Traumatología y Editor del Arthroscopy: The Journal of Arthroscopic and Related Surgery. El resto de los autores, no presentan ningún conflicto de interés ni reciben ningún beneficio personal o profesional directa o indirectamente en relación a este manuscrito.

\section{Bibliografía}

1 Haley CA, Zacchilli MA. Pectoralis major injuries: evaluation and treatment. Clin Sports Med 2014;33(04):739-756

2 Balazs GC, Brelin AM, Donohue MA, et al. Incidence Rate and Results of the Surgical Treatment of Pectoralis Major Tendon Ruptures in Active-Duty Military Personnel. Am J Sports Med 2016;44(07):1837-1843

3 White DW, Wenke JC, Mosely DS, Mountcastle SB, Basamania CJ. Incidence of major tendon ruptures and anterior cruciate ligament tears in US Army soldiers. Am J Sports Med 2007;35(08):1308-1314
4 ElMaraghy AW, Devereaux MW. A systematic review and comprehensive classification of pectoralis major tears. J Shoulder Elbow Surg 2012;21(03):412-422

5 Bak K, Cameron EA, Henderson IJ. Rupture of the pectoralis major: a meta-analysis of 112 cases. Knee Surg Sports Traumatol Arthrosc 2000;8(02):113-119

6 Tietjen R. Closed injuries of the pectoralis major muscle. J Trauma 1980;20(03):262-264http://www.ncbi.nlm.nih.gov/pubmed/7359 604. Accessed September 4, 2017

7 Pochini AdeC, Andreoli CV, Ejnisman B, Maffulli N. Surgical repair of a rupture of the pectoralis major muscle. BMJ Case Rep 2015; 2015:1-3

8 Connell DA, Potter HG, Sherman MF, Wickiewicz TL. Injuries of the pectoralis major muscle: evaluation with MR imaging. Radiology 1999;210(03):785-791

9 Chang ES, Zou J, Costello JM, Lin A. Accuracy of magnetic resonance imaging in predicting the intraoperative tear characteristics of pectoralis major ruptures. J Shoulder Elbow Surg 2016;25(03):463-468

10 Sherman SL, Lin EC, Verma NN, et al. Biomechanical analysis of the pectoralis major tendon and comparison of techniques for tendoosseous repair. Am J Sports Med 2012;40(08):1887-1894

11 Rabuck SJ, Lynch JL, Guo X, et al. Biomechanical comparison of 3 methods to repair pectoralis major ruptures. Am J Sports Med 2012;40(07):1635-1640

12 Fung L, Wong B, Ravichandiran K, Agur A, Rindlisbacher T, Elmaraghy A. Three-dimensional study of pectoralis major muscle and tendon architecture. Clin Anat 2009;22(04):500-508

13 Wolfe SW, Wickiewicz TL, Cavanaugh JT. Ruptures of the pectoralis major muscle. An anatomic and clinical analysis. Am J Sports Med 1992;20(05):587-593

14 Carrino JA, Chandnanni VP, Mitchell DB, Choi-Chinn K, DeBerardino TM, Miller MD. Pectoralis major muscle and tendon tears: diagnosis and grading using magnetic resonance imaging. Skeletal Radiol 2000;29(06):305-313

15 Sun Joo Lee MD, Jon A. Jacobson, MD, Sung-Moon Kim, MD, David Fessell, MD, Yebin Jiang, MD, PhD, Gandikota Girish, MD and Olaf Magerkurth M. Distal Pectoralis Major Tears. J Ultrasound Med 2013;32:2075-2081

16 Antosh IJ, Grassbaugh JA, Parada SA, Arrington ED. Pectoralis major tendon repairs in the active-duty population. Am J Orthop 2009;38(01):26-30

17 Butt U, Mehta S, Funk L, Monga P. Pectoralis major ruptures: a review of current management. J Shoulder Elbow Surg 2015;24 (04):655-662. Doi: 10.1016/j.jse.2014.10.024

18 Gregory JM, Klosterman EL, Thomas JM, et al. Suture technique influences the biomechanical integrity of pectoralis major repairs. Orthopedics 2015;38(09):e746-e752 